\title{
KLYSTRON POWER SPECIFICATIONS BASED ON TRANSIENT BEAM LOADING ANALYSIS IN DAMPING RINGS*
}

\author{
M.G. Minty, R.H. Siemann \\ Stanford Linear Accelerator Center, Stanford University, Stanford, CA 94309 USA
}

\begin{abstract}
A useful diagram is presented and used to study rf cavity voltage regulation in a damping ring or other circular accelerator where large beam currents are injected and extracted rapidly. With conventional feedback systems and high particle beam currents, the maximum beam current can be limited by the ability of the klystron to maintain constant gap voltage at high currents, low currents, or in the absence of beam. Techniques for storing high current beams are suggested when the maximum klystron output power is predetermined. Alternately, these techniques may be applied and used to specify the performance requirements of the klystron if the desired particle beam current and nominal rf cavity voltage are known.
\end{abstract}

\section{INTRODUCTION}

Experience $^{1,2}$ with high current beams and transient beam loading in the Stanford Linear Collider (SLC) damping rings has motivated studies of the rf system and beam stability with a nonlinear power source ${ }^{2}$. As beam currents in fast cycling accelerators are increased, the absence of beam is the dominant perturbation to the rf system, where slow tuner feedback loops around each cavity have insufficient bandwidth to track the changes. The beam may be absent due to machine repetition rate changes, due to time required between fills, or due to gaps between bunch trains. With standard feedback and direct rf feedback ${ }^{3}$ for regulation of the cavity voltage, the klystron output power can increase substantially when the beam is absent. While the behavior without beam is of little concern, missing pulses can leave the rf system out of regulation when the next pulse arrives. The beam can then become unstable with both the bunch length and the beam phase at extraction varying with time.

The phasor diagram corresponding to the generic circuit model of the beam cavity interaction is shown in Fig. 1. The current used in the equivalent circuit ${ }^{3,4} \vec{I}_{b}$ is $180^{\circ}$ out of phase with the actual beam current. The generator current $\vec{I}_{g}$ is summed with $\vec{I}_{b}$ to give the total cavity current $\vec{I}_{c}$. The beam current, or magnitude of $\vec{I}_{b}$, is twice the dc beam current: $I_{b}=2 I_{d c}$. The phase of the beam $\phi_{b}$ is measured with respect to the crest of the rf. The equilibrium value of $\phi_{b}$ is the synchronous phase $\phi_{s}$. The projection of $\vec{I}_{c}$ onto the cavity voltage is the shunt resistor current, $I_{0}=\frac{V_{c}}{R}$. The tuning angle is the angle between $\vec{I}_{c}$ and the cavity voltage $\vec{V}_{c}$ :

$$
\phi_{z}=\tan ^{-1}\left[2 Q \frac{\left(\omega_{0}-\omega_{r f}\right)}{\omega_{0}}\right],
$$

in which $Q$ and $\omega_{0}$ are the quality factor and resonance frequency of the cavity, respectively, while $\omega_{r f}$ is the accelerating

\footnotetext{
*Work supported by Department of Energy contract DE-AC03-76SF00515.
}

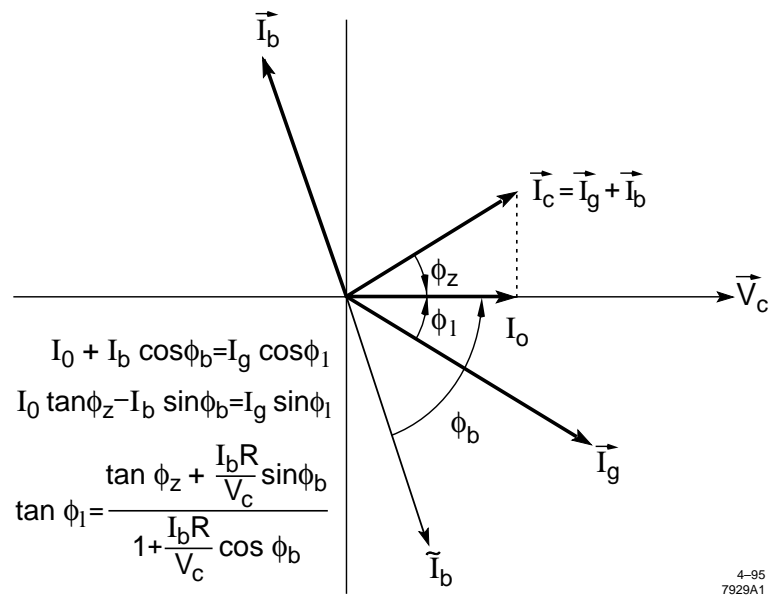

Figure. 1. Phasor diagram for a capacitive cavity and a beam above transition.

frequency. The angle between $\vec{I}_{g}$ and $\vec{V}_{c}$ is the loading angle, $\phi_{l}$.

\section{PARAMETER SPACE FOR VOLTAGE REGULATION}

A relationship between the beam current $\vec{I}_{b}$, generator power $P_{g}$, and cavity voltage $\vec{V}_{c}$ can be derived using the equations developed in reference 5 . The result is

$$
\begin{gathered}
I_{b}=\frac{V_{c}}{R \cos \phi_{z}}\left[-\cos \left(\phi_{z}-\phi_{b}\right)\right. \\
\left. \pm \sqrt{\cos ^{2}\left(\phi_{z}-\phi_{b}\right)+\left(\frac{8 R \beta_{c} P_{g}}{V_{c}^{2}\left(1+\beta_{c}\right)}\right) \cos ^{2} \phi_{z}-1}\right]
\end{gathered}
$$

in which $\beta_{c}$ is the cavity coupling coefficient. This expression remains valid in the presence of feedback loops. The parameter space for voltage regulation in the steady state is shown in Fig. 2 for the SLC damping rings. The solid curve bounding regions 1,2, and 3 shows Eq. (2) for a $60 \mathrm{~kW}$ klystron output power. In order to operate outside this region, either the klystron power would have to be increased or the gap voltage lowered. The curve with circles is a simulation ${ }^{2,4}$ result which takes into account the dynamics of the beam-cavity interaction in the presence of multiple feedback loops and a nonlinear power source. Region 3, which is bounded from above by Eq. (2) and below by the simulation results, is unstable against perturbations to the system.

The vertical line separating regions 1 and 2 represents a practical limit associated with missing pulses. Consider an rf current of magnitude $I_{b}=I_{m}$ or $I_{b}=I_{n}$. When this pulse is extracted, the loading angle becomes large and negative as the slow tuner feedback loops have insufficient bandwidth to track the change; 


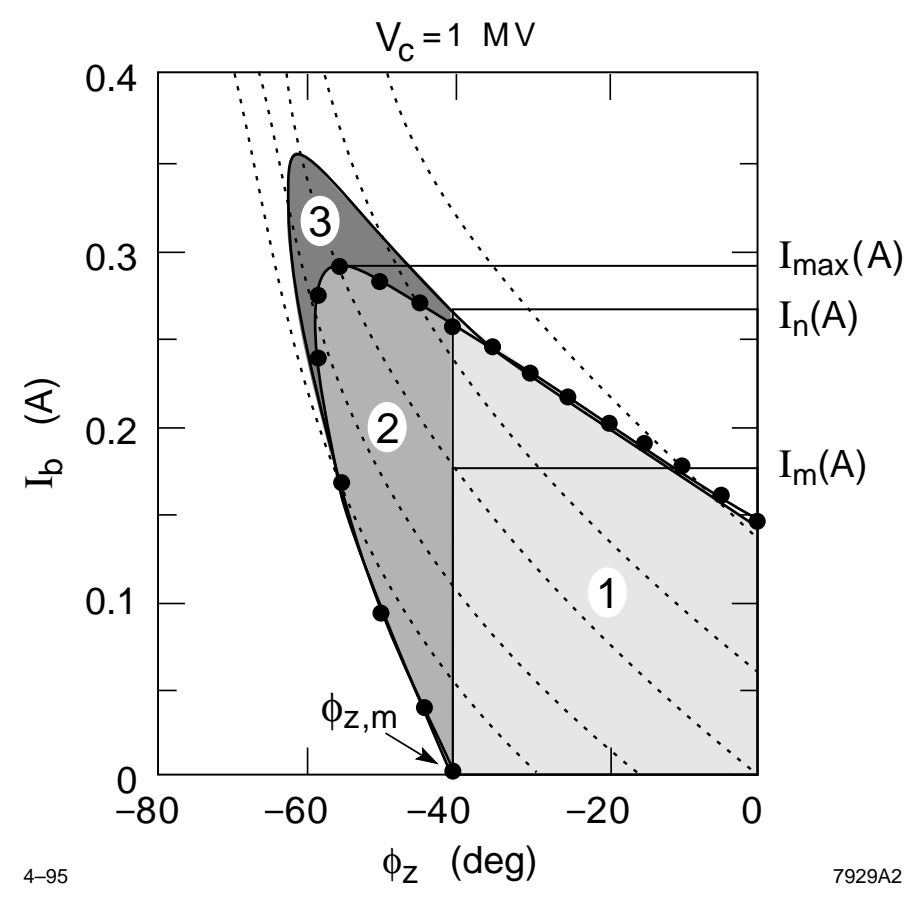

Figure. 2. Parameter space for voltage regulation subdivided into three characteristic regions. The dotted lines indicate contours of constant loading angle $\phi_{l}$. From left to right these are $\phi_{l}=-30^{\circ}$, $-15^{\circ}, 0^{\circ},+15^{\circ}$, and $+30^{\circ}$.

the operating point moves downward (towards $I_{b}=0$ ) on this vertical line. A voltage error related to the change in the beaminduced voltage is detected by the feedback loops that regulate the cavity voltage. If direct rf feedback is used or if the cavity and amplitude loop bandwidth are fast relative to the time during which the beam is absent, then the klystron is driven to larger output power in an attempt to compensate for this error. Because the gain of the amplitude feedback loops depends on the local slope of the klystron saturation curve, as the feedback drives the klystron harder, the gain of the loop is simultaneously decreasing. In particular, if the klystron is improperly limited, then a missing pulse or current jitter can lead to a situation of positive feedback.

\section{KLYSTRON POWER REQUIREMENTS}

Analysis of the available operating space for voltage regulation may be used to make an estimate of the required output power from a klystron. At high beam current and high cavity voltage, however, the estimate may be unrealistic. Methods of optimizing the stability of the rf system at high currents, while minimize the power requirements of the klystron will now be considered.

\section{A. Power Requirements with $\phi_{l}=0$}

A conservative estimate for the required klystron power may be determined from the missing pulse limit; for a properly matched cavity $\left(\phi_{l}=0\right)$ and known operating voltage, the klystron should deliver as much power as required such that the beam current is less than $I_{m}$. Rewriting Eq. (2),

$$
P_{g}=\frac{V_{c}^{2}\left(1+\beta_{c}\right)}{8 R \beta_{c} \cos ^{2} \phi_{z}}\left[\left(\frac{I_{b} R}{V_{c}}\right)^{2} \cos ^{2} \phi_{z}\right.
$$

$$
\left.+\frac{2 I_{b} R}{V_{c}} \cos \phi_{z} \cos \left(\phi_{z}-\phi_{b}\right)+1\right],
$$

which gives the required generator power in terms of the rf current $I_{b}$, the cavity voltage $V_{c}$, and the cavity tuning angle $\phi_{z}$. The tuning angle at $\phi_{l}=0$ and $I_{b}=I_{m}$ is

$$
\phi_{z, m}=-\tan ^{-1}\left(\frac{I_{m} R}{V_{c}} \sin \phi_{b}\right) .
$$

Substituting $\phi_{z, m}$ into (3) and putting $I_{b}=0$ gives the klystron power required to be stable against missing pulses when the nominal rf current is $I_{m}$ :

$$
P_{m}=\frac{V_{c}^{2}\left(1+\beta_{c}\right)}{8 R \beta_{c}}\left[1+\left(\frac{I_{m}}{I_{0}}\right)^{2} \sin ^{2} \phi_{b}\right], \text { with } \phi_{l}=0 .
$$

With beam, the klystron output power in the steady state is Eq. (3) evaluated at $I_{m}$, which is less than $P_{m}$.

\section{B. Power Requirements with Cavity Detuning $\left(\phi_{l} \neq 0\right)$}

By detuning the cavity to $\phi_{l}>0$, more beam current can be stored stably for the same maximum klystron power while maintaining stability against missing pulses. At $I_{b}$ just less than $I_{n}$, the klystron power with and without beam is approximately the same, however much of the output power however is reflected back into the isolator. From Eq. (2) the maximum current $I_{c}$ operable while maintaining stability against missing pulses results when the two terms in the square brackets are equal. Then

$$
I_{n}=-\frac{2 V_{c}}{R \cos \phi_{z}} \cos \left(\phi_{z}-\phi_{b}\right) \text {. }
$$

The klystron power required is Eq. (3) evaluated at $I_{b}=I_{c}$.

\section{Optimal Use of Klystron Power by RF Conditioning with} $\phi_{l}=0$

To achieve higher beam currents for the same maximum klystron output power, the klystron must be made to operate more efficiently. For operation in region 2 of Fig. 2, the hard limit due to missing pulses or absence of beam must be overcome. This may be accomplished by lowering the reference to the direct $\mathrm{rf}$ feedback loop in the case of a missing pulse. Highlighted in Fig. 3 are the voltage error $\Delta V$ and the phase error $\Delta \phi$ that result from an absent beam. Ideally, one might raise $\vec{V}_{g}$ when the beam is absent to equal the steady state cavity voltage with beam $\vec{V}_{c}$. If the klystron is power limited, however, this is impossible. Alternatively, the output of the amplitude loop can be lowered by changing the reference input during the time $I_{b}=0$. The output of the amplitude feedback loop is the reference for the direct $\mathrm{rf}$ feedback loop. Along $\phi_{l}=0$ the change in the cavity voltage setpoint required for correction is

$$
\Delta V=V_{c}+V_{b} \frac{\sin \left(\phi_{b}-\phi_{z}\right)}{\sin \phi_{z}} .
$$

The change in the phase reference setpoint required for correction is $\Delta \phi=\phi_{z}-\phi_{l}$. The corrections should be disabled at injection of the next pulse. More generally, transients arising from beam intensity fluctuations could be minimized by applying voltage and phase corrections which depend on the input current. In 


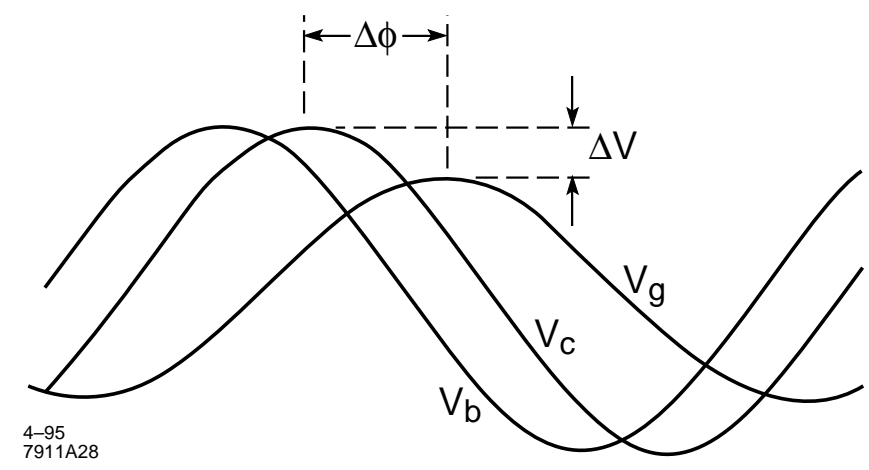

Figure. 3. Cavity voltages highlighting errors in the voltage $\Delta V$ and phase $\Delta \phi$ resulting from beam extraction.

practice the amount by which to lower the voltage setpoint would be to that value for which the control voltage from the amplitude feedback loop is unchanged by the change in beam current at injection. The amount by which to change the phase setpoint would be to minimize the change in the cavity voltage phase angle.

Simulation results using $\mathrm{rf}$ conditioning are shown in Fig. 4 for the case of a missing pulse. Without rf conditioning, the klystron operates well beyond the knee of the saturation curve during the time $I_{b}=0$. When the beam is injected, the gain of the amplitude and direct rf feedback loops are zero and the cavity voltage does not regulate. Poor regulation is also evidenced in the amplitude feedback loop control voltage which exhibits runaway during the time the beam is missing. With conditioning of the rf voltage, the voltage reference is reduced when the missing pulse is detected. The next pulse is anticipated and the reference is raised. During the time in which the beam was absent the klystron is brought out of saturation. The cavity voltage is then well regulated when the beam is injected.

\section{RF PARAMETER OPTIMIZATION}

As beam currents in damping rings are increased, specifications for the klystron power and feedback loop parameters should involve analysis of the effects of transient loading on the rf system. Using the plot of the parameter space, effects arising from the largest possible transient, that of pulse extraction, may be analyzed. Equation 2 shows that the available operating area changes as a function of cavity voltage, cavity impedance, cavity coupling, klystron power, and synchronous phase. Tradeoffs between klystron output power and these other parameters can be considered in the design of the rf system.

\section{References}

[1] M.G. Minty et al., WAB12 1995 PAC.

[2] M. Minty and R.H. Siemann, RAP10 1995 PAC.

[3] F. Pederson, IEEE Tran. on Nucl. Sci., NS-32, No. 3 (1985) 2138.

[4] M.G. Minty, R.H. Siemann, "Heavy Beam Loading in Storage Ring RF Systems," to be submitted to Nucl. Instr. and Methods.

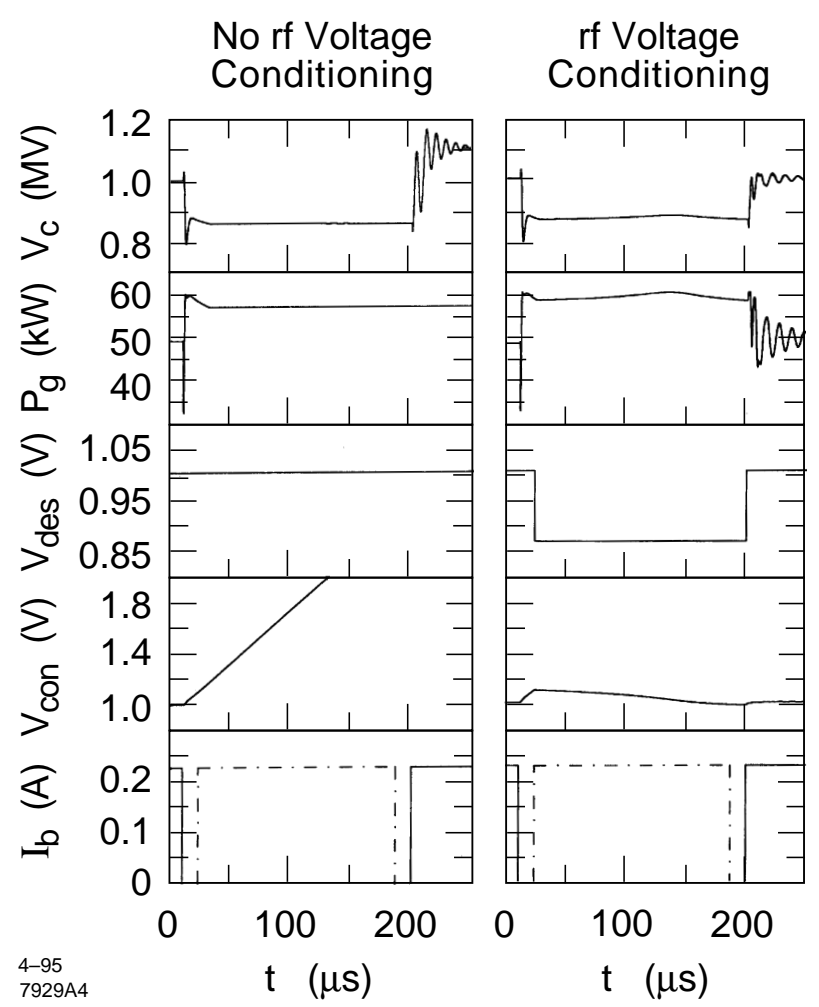

Figure. 4. Cavity regulation and voltage transient reduction in the SLC with and without rf conditioning in the event of a missing pulse. Plotted as a function of time are the cavity voltage $V_{c}$, the klystron output power $P_{g}$, the reference input to the amplitude feedback loop $V_{d e s}$, the amplitude feedback control voltage $V_{c o n}$, and the rf current $I_{b}$. The dot-dashed curve in $I_{b}$ indicates the missing pulse.

[5] P. Wilson, "High Energy Electron Linacs; Application to Storage Ring RF Systems and Linear Colliders," in Phys. of High-Energy Part. Acc., AIP Conf. Proc. No. 87 (AIP, NY 1982), pp.450-563. SLAC-PUB-2884 (1982). 